\title{
A Cost-Utility and Cost-Effectiveness Analysis of Different Oral Antiviral Medications in Patients With HBeAg-Negative Chronic Hepatitis B in Iran: An Economic Microsimulation Decision Model
}

\author{
Khosro Keshavarz, ${ }^{1}$ Abbas Kebriaeezadeh, ${ }^{2}$ Seyed Moayed Alavian, ${ }^{3}$ Ali Akbari Sari, ${ }^{4}$ Mohsen Rezaei \\ Hemami, ${ }^{5}$ Farhad Lotfi, ${ }^{1}$ Amir Hashemi Meshkini, ${ }^{2}$ Mehdi Javanbakht, ${ }^{6}$ Maryam Keshvari, ${ }^{7}$ and \\ Shekoufeh Nikfar ${ }^{2, *}$ \\ ${ }^{1}$ Health Human Resource Research Center, School of Management and Information Sciences, Shiraz University of Medical Sciences, Shiraz, IR Iran \\ ${ }^{2}$ Department of Pharmacoeconomics and Pharmaceutical Administration, Faculty of Pharmacy, Tehran University of Medical Sciences, Tehran, IR Iran \\ ${ }^{3}$ Baqiyatallah Research Center for Gastroenterology and Liver Diseases, Baqiyatallah University of Medical Sciences, Tehran, IR Iran \\ ${ }^{4}$ Department of Health Management and Economics, Tehran University of Medical Sciences, Tehran, IR Iran \\ ${ }^{5}$ Institute of Health \& Wellbeing, Health Economics \& Health Technology Assessment, University of Glasgow, Glasgow, United Kingdom \\ ${ }^{6}$ Health Economics Research Unit, Institute of Applied Health Sciences, University of Aberdeen, Aberdeen, UK \\ ${ }^{7}$ Blood Transfusion Research Center, High Institute for Research and Education in Transfusion Medicine, Tehran, IR Iran \\ "Corresponding author: Shekoufeh Nikfar, Department of Pharmacoeconomics and Pharmaceutical Administration, Faculty of Pharmacy, Tehran University of Medical \\ Sciences, Tehran, IR Iran. Tel: +98-09123271200, Fax: +98-2188611883, E-mail: shekoufeh.nikfar@gmail.com
}

Received 2016 February 28; Revised 2016 July 02; Accepted 2016 August 07.

\begin{abstract}
Background: Although hepatitis B infection is the major cause of chronic liver disease in Iran, no studies have employed economic evaluations of the medications used to treat Iranian patients with chronic hepatitis B (CHB). Therefore, the cost-effectiveness of the different treatment options for this disease in Iran is unknown.

Objectives: The aim of this study was to compare the cost utility and cost-effectiveness of medication strategies tailored to local conditions in patients with HB e antigen (HBeAg)-negative CHB infection in Iran.

Methods: An economic evaluation of the cost utility of the following five oral medication strategies was conducted: adefovir (ADV), lamivudine (LAM), ADV + LAM, entecavir (ETV), and tenofovir (TDF). A Markov microsimulation model was used to estimate the clinical and economic outcomes over the course of the patient's lifetime and based on a societal perspective. Medical and nonmedical direct costs and indirect costs were included in the study and life-years gained (LYG) and quality-adjusted life-years (QALY) were determined as measures of effectiveness. The results are presented in terms of the incremental cost-effectiveness ratio (ICER) per QALY or LYG. The model consisted of nine stages of the disease. The transition probabilities for the movement between the different stages were based on clinical evidence and international expert opinion. A probabilistic sensitivity analysis (PSA) was used to measure the effects of uncertainty in the model parameters.

Results: The results revealed that the TDF treatment strategy was more effective and less costly than the other options. In addition, TDF had the highest QALY and LYG in the HBeAg-negative CHB patients, with 13.58 and 21.26 (discounted) in all comparisons. The PSA proved the robustness of the model results. The cost-effectiveness acceptability curves showed that TDF was the most cost-effective treatment in 59\% - 78\% of the simulations of HBeAg-negative patients, with WTP thresholds less than \$14010 (maximum WTP per QALY).

Conclusions: The use of TDF in patients with HBeAg-negative CHB seemed to be a highly cost-effective strategy. Compared with the other available medication options, TDF was the most cost-saving strategy. Thus, TDF may be the best option as a first-line medication. Patients can also be switched from other medications to TDF.
\end{abstract}

Keywords: Chronic Hepatitis B, Cost-Utility and Cost-Effectiveness Analysis, Oral Antiviral Therapy, Markov Microsimulation Model, Probabilistic Sensitivity Analysis

\section{Background}

Hepatitis B remains a serious health problem worldwide, as it is the most common and serious form of hep- atitis and liver disease (1). People with the disease have an increased risk of mortality and morbidity (2). In addition, treatment costs are high, especially in developing countries (2). Currently, an estimated 350 million people 
worldwide have chronic $\mathrm{HB}$ (CHB) infection, which results in about 500,000 to 1.2 million deaths per year $(3,4)$. In Iran, according to the latest statistical data released by the office for the prevention of viral hepatitis in the ministry of health and medical education, the prevalence of hepatitis B in the country was estimated to be about $1.5 \%(5,6)$. Given that the population of Iran was about 78 million in 2014 (7), the total number of chronic HB virus (HBV) carriers was estimated to be 1,170,000 people (5). The genotype $\mathrm{D}$ is the predominant genotype of hepatitis in the country $(8,9)$, and more than $70 \%$ of cases are HB e antigen (HBeAg) negative (10).

Given the mortality rate among untreated CHB patients $(20 \%-25 \%)(11)$, long-term and severe outcomes of CHB infection, and its complicated pattern of management, timely diagnosis and treatment are critical to prevent patients progressing to the severe stages of the disease. Although medication therapy cannot cure CHB, except in rare cases (11), early treatment can significantly prevent the progress of severe liver failure and reduce mortality (12).

Oral treatments (oral nucleos(t)ide analogues) are preferred as the first-line therapy for most genotypes of the disease, as they have fewer side effects compared with interferon (12). The goal of drug therapy for CHB patients is to improve their quality of life and survival by preventing progression of the disease to more severe stages, such as liver cirrhosis and liver cancer. At present, five oral medication strategies are used to treat hepatitis in Iran: adefovir (ADV), lamivudine (LAM), ADV + LAM, entecavir (ETV), and tenofovir (TDF). The use of ETV is less common because of its high cost and the fact that it is not indexed in the Iran Drug List. However, to evaluate the cost-effectiveness of all oral drugs in Iran, ETV was included in the studied medication strategies.

Economic evaluation studies published in recent years showed that TDF was more cost effective than other drugs (12-16). However, most of the published economic evaluation studies on CHB treatment have been carried out in the U.S., Europe, and Southeast Asian countries. In contrast, in the Eastern Mediterranean region, specifically Iran, no studies have used economic evaluation models in the assessment of medication therapy protocols for patients with hepatitis B.

Accordingly, due to the lack of sufficient scientific evidence, the best strategy for the treatment of hepatitis B patients in Iran is unclear. The provision of accurate data on both the economic and clinical impact of HBV infection can help policymakers to make informed decisions to provide better health and medical treatment services for these patients. A number of factors necessitated this study. These included the high prevalence of $\mathrm{HBV}$, which accounts for the majority of hepatitis infections in the country, its role as the major cause of cirrhosis and liver cancer $(5,17,18)$, the need to identify optimum hepatitis B strategies tailored to local conditions, and the absence of economic evaluation studies on the disease in Iran and the Eastern Mediterranean region.

\section{Objectives}

This study aimed to assess the cost utility and costeffectiveness of various medication strategies for HBeAgnegative $\mathrm{CHB}$ from a societal perspective to determine the most appropriate protocol for treating $\mathrm{HBV}$ infection in Iran.

\section{Methods}

\subsection{Overview}

This study consisted of an economic evaluation of the cost utility of five oral medication strategies: ADV, LAM, ADV + LAM, ETV, and TDF. Unlike other studies in the field of hepatitis, we used a Markov microsimulation model to identify economic and clinical outcomes of HBV infection, which requires a long-term follow up due to the chronic and recurring nature of the infection (12). The outcome measures used in the model included life-years gained (LYG), quality-adjusted life-years (QALY), and the costs of the disease and treatment strategy in each state. For extracting costs, a questionnaire was prepared, and its validity and reliability were tested. As this study adopted a societal perspective, both medical and nonmedical costs (direct and indirect) were evaluated. The time horizon of the study was the patient's lifetime, with one-year cycles, lasting a total of 35 years. In common with other published economic evaluation studies $(12,13)$ and in accordance with the opinions of experts, the model was run with one-year cycles to cope with the nature of the changes in the various stages of the disease. As the duration of the model in this study was more than one year, the costs and effects of the medication therapy for patients were calculated for one year in 2014. These were discounted during the patient's lifetime, with an annual rate of $7.2 \%$ (19) and $3 \%(20)$, respectively. These discount rates have been applied in previous economic evaluation studies published in Iran (21). Excel and Tree Age software, V. 2011 were used to analyze the data.

\subsection{Study Population and Treatment Regimens}

The sample size of this study consisted of 155 patients with HBeAg-negative CHB. The sample size was calculated based on the statistical sample of the present study and the 
profiles registered in the studied centers. Therefore, the characteristics of the patients matched those of the real population of patients who were referred to the research center of gastroenterology and hepatology in Baghiatollah University and Baghiatollah hospital and Nemazee hospital in Shiraz. The centers were selected using purposive sampling because they are referral centers for hepatitis in the country, and people from different parts of the country are referred to these centers.

The mean age of the patients in the sample was $40 \mathrm{y}$, and $67 \%$ of the study group were males.

At the end, a microsimulation analysis of a hypothetical cohort population of 10000 individuals was carried out.

\subsection{Inclusion and Exclusion Criteria}

The inclusion criteria were as follows: infected with $\mathrm{CHB}$, a biopsy- or fibroscan-confirmed diagnosis of $\mathrm{CHB}$, received the medications evaluated in the study for at least one year, and had a record. The exclusion criteria were patients with acute hepatitis B, HBeAg-positive CHB and newly diagnosed patients, other disease states, and other types of hepatitis.

The study was approved by the ethics committee of the Pharmaceutical Sciences Research Center of Tehran University of Medical Sciences.

The medication therapy regimens included in the model were based on the guide for the treatment of chronic hepatitis $B(22)$. The regimens were as follows: ADV (10 mg daily), LAM (100 mg daily), TDF (300 mg daily), ETV (0.5 mg daily), and a combination of ADV and LAM. All the medications were taken orally.

\subsection{Switching Medications}

Based on clinical evidence worldwide (23-25) and in Iran (26) on the development of drug resistance, side effects of drugs, or futility of drugs in some cases, medication regimens may need to be altered to enable the treatment to be continued and prevent disease progression in patients.

This study used Tree Age software to design and evaluate a medication-switching model. The medications were switched in cases of drug resistance, side effects, and no response to the initial treatment. According to published studies, the development of resistance was higher with ADV and LAM than other drugs (27-30). However, as gastroenterology and hepatology specialists in Iran often prescribe a combination of two medications (ADV + LAM and TDF), withdrawal was designed only for these two medications, and the possibilities of resistance were considered only for this state.
Concerning the option to switch to other medications, according to the existing registry of gastroenterology and hepatology Centers, 37\% and 63\% of patients resistant to LAM, respectively, switched to TDF and ADV + LAM; moreover, $48 \%$ and $52 \%$ of patients resistant to ADV, respectively, switched to TDF and ADV + LAM.

\subsection{Model Structure}

Figure 1 presents a schematic diagram of the Markov model of the studied sample and for a hypothetical cohort population of 10000 HBeAg-negative patients aged over 18 y.

As shown in the diagram, CHB patients receiving antiviral drugs can have a recurring transition to different disease states. The specified states and their changes were determined based on evidence from other studies and the opinions of experts. Patients in all stages of the disease can develop hepatocellular carcinoma (HCC) and die. The patients included in the initial distribution of the Markov microsimulation model were selected in proportion to a real sample of patients with $\mathrm{CHB}$.

\subsection{Key Modeling Assumptions}

Based on the available clinical evidence and the results of a review of the medical records of the patients included in the study, the resistance to ETV and TDF drugs was assumed to be zero.

In the first five years, the drug resistance rates for both LAM and ADV were considered to be variable (31). In subsequent years, they were determined based on the values in the fifth year.

As clinical evidence suggests that combination therapy may not result in more effective therapy (32-34) and due to the lack of published data, it was assumed that the transition probabilities between some of the health states were equal to the most effective component of the combination medication shown in Table 1.

It was assumed that nucleos(t)ides had no effect on mortality in patients with HCC and decompensated cirrhosis (DC).

It was assumed that the side effects associated with nucleos(t)ides had no impact on cost, quality of life, and mortality. Nevertheless, the cost of monitoring renal failure was considered in the cost analysis of the model.

For some transitions, such as hepatitis B surface antigen (HBsAg) to HCC, CHB to HBsAg, and CHB to UnHBV DNA to HCC, data on the individual drugs were not available. In these cases, it was assumed that all treated patients had an equal chance of improvement or disease progression, irrespective of the type of medication used. 


\section{HBeAg Negative CHB}

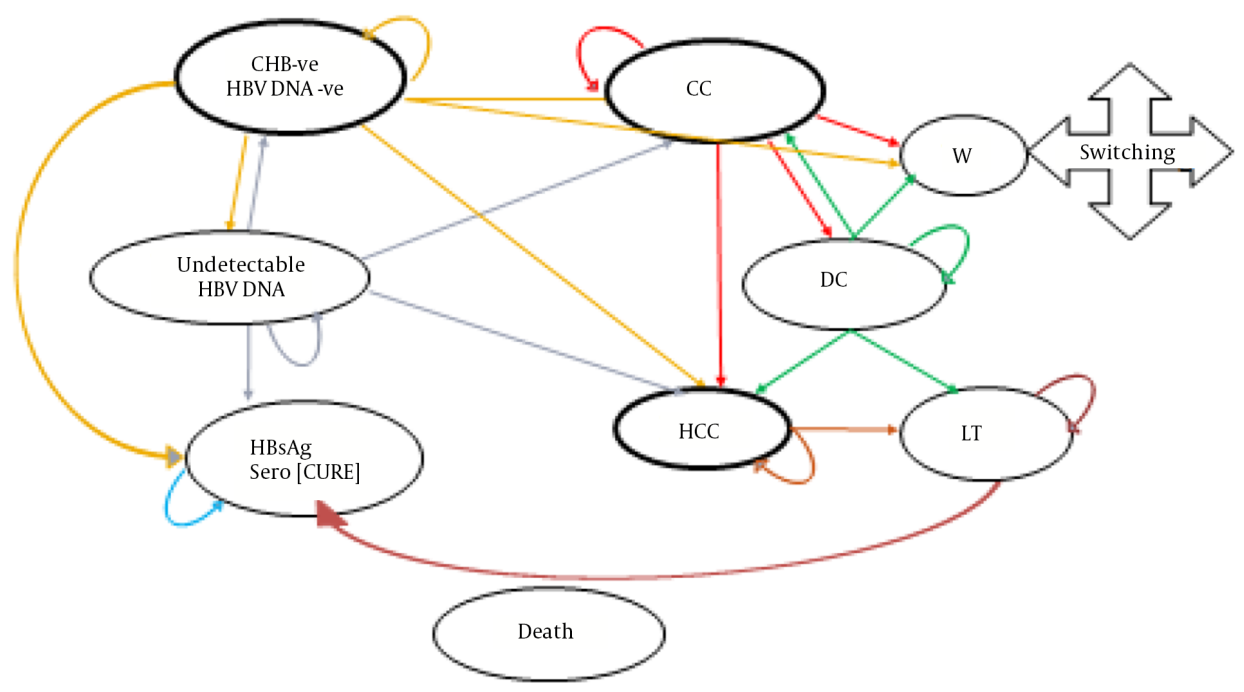

Figure 1. Decision Analysis Model to Evaluate the Cost-Effectiveness of Treatment Strategies for HBeAg-Negative CHB Patients

\subsection{Transitional Probabilities}

Tables 1 and 2 present the transitional probabilities for all the parameters included in the model. Due to the absence of data from Iranian studies, especially on the clinical efficacy of different medication protocols used for patients with $\mathrm{CHB}$, most of the transitional probabilities between health states were extracted from international studies and evidence on hepatitis B (12, 13, 31-49).

The mortality rate for the general population was extracted from life tables for 2012 and analyzed separately by age and sex (50). To determine the rate of deaths due to hepatitis $\mathrm{B}$, the standardized mortality ratio reported in published articles was used (51). Some probabilities entered in the model were based on the medical records of patients at the centers included in the study.

As the data on clinical efficacy were reported as rates in some articles, they were converted to probabilities using the following Formula 1 and then entered in the model:

$p=1-\exp ^{(-r t)}$

Where $\mathrm{p}$ is the probability, $\mathrm{r}$ is the instantaneous rate, and $\mathrm{t}$ is the time (52).

\subsection{Health Outcomes}

Health outcomes were specified in terms of LYG and QALY. Utility scores were obtained using EQ-5D questionnaires administered via face-to-face interviews conducted with 155 HBeAg-negative CHB patients in 2014. Related complications, such as compensated cirrhosis (CC), DC, and HCC, were recorded. The interviewees in the study consisted of outpatients referred to the research center for gastroenterology and hepatology in Baghiatoallh University in Tehran (the capital of Iran and a hepatitis referral center) and inpatients admitted to two major hospitals in Tehran and Shiraz. These hospitals are the main treatment centers for liver transplantation patients.

After completing the EQ-5D questionnaire, for the first time, we tried to consider the national values of Iran that were obtained in a separate study by Goudarzi (53) using time trade-off (TTO), and we changed the 5-digit codes of the questionnaire into numerical utility (Table 3). In addition, in a separate calculation using values reported in a U.K. study, the 5-digit codes were changed again into numerical utility and used in the sensitivity analysis of the model.

\subsection{Treatment Costs}

The cost analysis method used in a study performed in 2105 was applied (5). The costs of each medication strategy in each state for the treatment of CHB, CC, DC, and HCC were calculated, as shown in Table 2. Data on the direct and indirect costs were extracted from both specialists' and patients' views and a review of inpatient and outpatient medical records. 
Table 1. Input Parameters Used in the Economic Microsimulation Decision Model: HBeAg-Negative CHB

\begin{tabular}{|c|c|c|c|c|}
\hline Variable & Mean & Dist & Lower Values & Upper Values \\
\hline Prob CHB to HCC with LAM & 0.048 & Beta & 0.02 & 0.09 \\
\hline Prob CHB to HCC with TDF & 0.012 & Beta & 0.008 & 0.015 \\
\hline Prob CHB to HCC with ADV + LAM & 0.048 & Beta & 0.02 & 0.09 \\
\hline Prob CHB to HCC with ETV & 0.014 & Beta & 0.008 & 0.02 \\
\hline Prob CHB to HCC with ADV & 0.048 & Beta & 0.02 & 0.09 \\
\hline Prob CC to DC with ADV & 0.027 & Beta & 0.014 & 0.054 \\
\hline Prob CC to DC with LAM & 0.057 & Beta & 0.031 & 0.099 \\
\hline Prob CC to DC with ETV & 0.034 & Beta & 0.0063 & 0.0623 \\
\hline Prob CC to DC with TDF & 0.019 & Beta & 0.014 & 0.054 \\
\hline Prob CC to DC with ADV + LAM & 0.027 & Beta & 0.014 & 0.054 \\
\hline Prob CC to HCC with ADV & 0.039 & Beta & 0.02 & 0.071 \\
\hline Prob CC to HCC with LAM & 0.041 & Beta & 0.0092 & 0.0728 \\
\hline Prob CC to HCC with ADV + LAM & 0.039 & Beta & 0.02 & 0.071 \\
\hline Prob CC to HCC with TDF & 0.016 & Beta & 0.01 & 0.056 \\
\hline Prob CC to HCC with ETV & 0.016 & Beta & 0.01 & 0.056 \\
\hline Prob HCC to IT & 0.008 & Beta & 0.00 & 0.031 \\
\hline Prob DC to LT & 0.010 & Beta & 0.00 & 0.2 \\
\hline Prob UnHBV DNA to CHB with TDF & 0.075 & Beta & 0.036 & 0.114 \\
\hline Prob CHB to CC with ADV & 0.03 & Beta & 0.00 & 0.0663 \\
\hline Prob CHB to CC with ADV+ LAM & 0.02 & Beta & 0.0055 & 0.0345 \\
\hline Prob CHB to CC with ETV & 0.007 & Beta & 0.00 & 0.0157 \\
\hline Prob CHB to CC with LAM & 0.02 & Beta & 0.0055 & 0.0345 \\
\hline Prob CHB to CC with TDF & 0.006 & Beta & 0.00 & 0.0169 \\
\hline Prob DC to HCC with ADV & 0.065 & Beta & 0.022 & 0.074 \\
\hline Prob DC to HCC with ADV + LAM & 0.025 & Beta & 0.022 & 0.074 \\
\hline Prob DC to HCC with ETV & 0.049 & Beta & - & - \\
\hline Prob DC to HCC with LAM & 0.066 & Beta & 0.02 & 0.111 \\
\hline Prob DC to HCC with TDF & 0.029 & Beta & 0.01 & 0.063 \\
\hline Prob CHB to UnHBV DNA with ADV & 0.489 & Beta & 0.258 & 0.771 \\
\hline Prob CHB to UnHBV DNA with TDF & 0.938 & Beta & 0.796 & 0.993 \\
\hline Prob CHB to UnHBV DNA with ADV+ LAM & 0.489 & Beta & 0.258 & 0.771 \\
\hline Prob CHB to UnHBV DNA with ETV & 0.730 & Beta & 0.573 & 0.877 \\
\hline Prob CHB to UnHBV DNA with LAM & 0.381 & Beta & 0.337 & 0.424 \\
\hline Prob UnHBV DNA to $C C$ & 0.005 & Beta & 0.00 & 0.013 \\
\hline Prob UnHBV DNA to CHB & 0.210 & Beta & 0.145 & 0.275 \\
\hline Prob UnHBV DNA to CHB with ETV & 0.075 & Beta & 0.036 & 0.114 \\
\hline Prob UnHBV DNA to HBsAg & 0.018 & Beta & 0.003 & 0.013 \\
\hline Prob UnHBV DNA to HCC & 0.005 & Beta & 0.002 & 0.015 \\
\hline Prob HBsAg to HCC & 0.005 & Beta & 0.0004 & 0.041 \\
\hline Prob CHB to HBsAg & 0.004 & Beta & 0.002 & 0.009 \\
\hline Prob CHB to UnHBV DNA-resistance to LAM or ADV & 0.306 & Beta & 0.089 & 0.701 \\
\hline Prob CHB to UnHBV DNA-resistance to LAM or ADV & 0.539 & Beta & 0.073 & 0.967 \\
\hline
\end{tabular}

Abbreviations: HBeAg, hepatitis B e antigen; CHB, chronic hepatitis B; Prob, probability; CC, compensated cirrhosis; DC, decompensated cirrhosis; HCC, hepatocellular carcinoma; Sero, seroconversion; LT, liver transplantation; W, withdrawal; LAM, lamivudine (100 mg) TDF, tenofovir (300 mg); ETV, entecavir (0.5 mg); ADV, adefovir (10 mg); HBsAg, hepatitis B surface antigen; Dist, distribution.

The costs (in US\$) were based on 2014 tariffs. The exchange rate was 26,668 rials per dollar (54). To enable international comparisons, the costs were changed to international dollars using a purchasing power parity (PPP) \$ exchange rate of 8565.41 rials per $1 \$(55)$.

\subsection{Cost-Effectiveness Analysis}

Based on the results of the aforementioned stages, a model was designed using Tree Age software, and the extracted data were entered into the model. Considering the time horizon of the study, the values for the costs, effectiveness, and cost-effectiveness were calculated as monetary currencies, QALY, and cost per QALY, respectively, for all the 
Table 2. Input Parameters Used in the Economic Evaluation Model: HBeAg Negative

\begin{tabular}{|c|c|c|c|c|}
\hline Variable & Mean & Dist & Lower Values & Upper Values \\
\hline Cost of CHB with ADV & 4256 & Gamma & 3405 & 5107 \\
\hline Cost of CHB with ADV + LAM & 4334 & Gamma & 3684 & 4970 \\
\hline Cost of CHB with ETV & 15496 & Gamma & 13800 & 18000 \\
\hline Cost of CHB with LAM & 4206 & Gamma & 3800 & 5000 \\
\hline Cost of CHB with TDF & 4922 & Gamma & 4100 & 5600 \\
\hline Cost of CC with ADV & 5280 & Gamma & 4224 & 6337 \\
\hline Cost of CC with ADV + LAM & 5358 & Gamma & 4600 & 6100 \\
\hline Cost of CC with ETV & 16514 & Gamma & 14500 & 18500 \\
\hline Cost of CC with LAM & 5231 & Gamma & 4500 & 6000 \\
\hline Cost of CC with TDF & 5944 & Gamma & 5200 & 6700 \\
\hline Cost of DC with ADV & 22476 & Gamma & 17980 & 26970 \\
\hline Cost of DC with ADV + LAM & 22554 & Gamma & 18900 & 26000 \\
\hline Cost of DC with ETV & 33709 & Gamma & 28000 & 40000 \\
\hline Cost of DC with LAM & 22426 & Gamma & 19000 & 26000 \\
\hline Cost of DC with TDF & 23139 & Gamma & 19000 & 27000 \\
\hline Cost of HCC with ADV & 40671 & Gamma & 33350 & 47990 \\
\hline Cost of HCC with ADV + LAM & 40749 & Gamma & 33400 & 48000 \\
\hline Cost of HCC with ETV & 51992 & Gamma & 45000 & 58000 \\
\hline Cost of HCC with LAM & 40621 & Gamma & 34000 & 47000 \\
\hline Cost of HCC with TDF & 41509 & Gamma & 33000 & 50000 \\
\hline Utility CHB & 0.83 & Beta & 0.68 & 0.85 \\
\hline Utility CC & 0.71 & Beta & 0.64 & 0.75 \\
\hline Utility DC & 0.48 & Beta & 0.40 & 0.53 \\
\hline Utility HCC & 0.51 & Beta & 0.27 & 0.59 \\
\hline Utility Sero & 0.95 & Beta & 0.90 & 0.95 \\
\hline Utility Un DNA & 0.83 & Beta & 0.77 & 0.85 \\
\hline
\end{tabular}

Abbreviations: HBeAg, hepatitis B e antigen; ADV, adefovir; LAM, lamivudine; ETV, entecavir; TDF, tenofovir.

interventions and their incremental cost-effectiveness ratio (ICERs) were estimated and compared using the following Formula 2 (56):

$$
\begin{aligned}
& \text { ICER } R_{T D F v \text { s.other antivirals }} \\
& =\frac{\text { Cost }_{T D F}-\text { Cost }_{\text {other antivirals }}}{Q A L Y / L Y G_{T D F}-Q A L Y / L Y G_{\text {other antivirals }}}
\end{aligned}
$$

\subsection{Uncertainty Analysis}

In this study, PSA was performed to evaluate the effects of uncertainty on the parameters included in the model. The probability distributions of all the parameters entered into the model are presented in Tables 1 and 2. A second-order Monte Carlo simulation was performed using 5000 trials to analyze the probabilistic sensitivity. The PSA results are presented using cost-effectiveness acceptability curves and a scatter plot of the incremental costeffectiveness. Due to the lack of an explicit WTP threshold in Iran, based on the proposal of the world health organization for developing countries, a WTP per every QALY of one to three times the gross domestic product per capita was used (57). In Iran, the value in 2014 when its threefold value was about $\$ 14010$ was $\$ 4670$ dollars. 
Table 3. Comparison of the Cost-Effectiveness of the Five Drug Strategies in Patients With HBeAg-Negative CHB Based on the Number of QALY Using the Markov Microsimulation Model

\begin{tabular}{lccccccc}
\hline Strategy & $\begin{array}{c}\text { Total } \\
\text { Cost } \\
\text { (USD) }\end{array}$ & LYG & QALY & $\begin{array}{c}\text { Incr. } \\
\text { Cost } \\
\text { (USD) }\end{array}$ & $\begin{array}{c}\text { Incr. } \\
\text { QALY }\end{array}$ & $\begin{array}{c}\text { ICER } \\
\text { Per } \\
\text { QALY }\end{array}$ & Subset \\
\hline -ve & -ve & -ve & - ve & - ve & -ve & \\
\hline TDF & 47,257 & 21.26 & 13.60 & 0 & 0 & 0 & Dominated \\
\hline LAM & 64,096 & 17.69 & 10.93 & 16,839 & -2.67 & -6300 & Dominated \\
\hline ADV & 69,510 & 18.59 & 11.51 & 22,253 & -2.09 & - & Dominated \\
\hline $\begin{array}{l}\text { Lam } \\
+ \text { Adv }\end{array}$ & 73,896 & 18.31 & 11.34 & 26,639 & -2.27 & -11743 & Dominated \\
\hline ETV & 139,532 & 20.92 & 13.47 & 92,275 & -0.13 & - & Dominated \\
\hline
\end{tabular}

Abbreviations: HBeAg, hepatitis B e antigen; Incr., incremental, ICER, incremental cost-effectiveness ratio; LYG, life-years gained; QALY, quality-adjusted lifeyears; TDF, tenofovir; LAM, lamivudine; ADV, adefovir; ETV, entecavir.

\section{Results}

\subsection{Base-Case Analysis}

In this study, 155 HBeAg-negative CHB patients who were referred to selected centers were enrolled in the study and classified by age, sex, and duration of disease in the initial model. The mean age of the total sample was $40 \mathrm{y}, 67 \%$ of the sample were males, and 33\% were females.

The results of the cost-effectiveness analysis obtained using the Markov microsimulation model and based on QALY and LYG showed that TDF was less expensive and more effective than the other medication strategies. Hence, TDF was the most cost-effective of the medication strategies (ADV, ETV, and LAM and ADV + LAM) (Figure 2).

Table 3 presents the cost-effectiveness (QALY and LYG), incremental costs, incremental effectiveness, and ICER, as well as the ratings and status of domination of the five drug therapy strategies in patients with HBeAg-negative CHB. As shown in the table, compared with TDF, the other strategies (LAM, ADV, ADV + LAM, and ETV) were associated not only with lower QALY and LYG but also with higher additional costs. During a patient's lifetime, compared with the alternatives, TDF had the lowest cost and highest expected QALY and LYG. ETV had the highest cost per every unit of QALY and LYG when compared with the other strategies.

\subsection{Uncertainty Analysis}

In this study, PSA was used to measure the effects of uncertainty in the parameters on the results of the model. Using 5000 trials, a second-order Monte Carlo simulation and PSA were conducted. The uncertainty of the results based on the range of WTP thresholds, cost-effectiveness acceptability curves, and a scatter plot of the incremental cost-effectiveness are presented in Figures 3 and 4.

An acceptability curve is one of the best curves for planning and policymaking. It can help policymakers and health system planners determine the cost-effectiveness of every intervention per payment. The results of the acceptability curves showed that TDF was the most cost-effective treatment strategy in $59 \%-78 \%$ of simulations for thresholds below $\$ 14010$ for patients with HBeAg-negative disease (Figure 3).

By showing what percentage of points is located in the accepted area (below the threshold), scatter plots present more detailed information for comparisons between single drugs. The scatter plot results are presented only for TDF compared with the alternatives. As compared with LAM, ADV, ETV, and ADV + LAM, the results demonstrated that TDF was in an acceptable area below the threshold in $85 \%, 84 \%, 62 \%$, and $83 \%$ of cases in patients with HBeAgnegative disease. Hence, it was the best of the other alternatives and the most cost-effective strategy (Figure 4). Therefore, the results of the sensitivity analysis did not change the status of TDF as the most effective treatment. This finding is an indication of the robustness of the results of the study.

\section{Discussion}

This is the first comprehensive economic evaluation study of hepatitis B patients in Iran and the Eastern Mediterranean region. The study aimed to evaluate oral nucleos(t)ide analogue strategies for HBeAg-negative CHB and its related complications from a pharmacoeconomic societal perspective.

In the present study, the ICERs based on QALY and LYG in patients with $\mathrm{HBeAg}$-negative $\mathrm{CHB}$ showed that of the five therapeutic strategies (ADV, LAM, ADV + LAM, ETV, and TDF) used in Iran, TDF was more effective and less expensive. This finding is in line with the results of He et al.'s study (12) and Tantai et al.'s study (58). Despite some minor differences, the results of this study are also consistent with those of previously published economic evaluation studies of HBV, such as the CUA study by Dakin et al. in the U.K. (13) and Buti et al. 's study (56) in Spain. The results of the present study demonstrated that TDF in patients with HBeAg-negative CHB had the lowest expected cost and highest expected QALY and LYG in a lifetime span. The results of this study are consistent with those of Tantai et al. (58).

The results of the PSA revealed that TDF was less expensive and more effective than the other drug therapy 


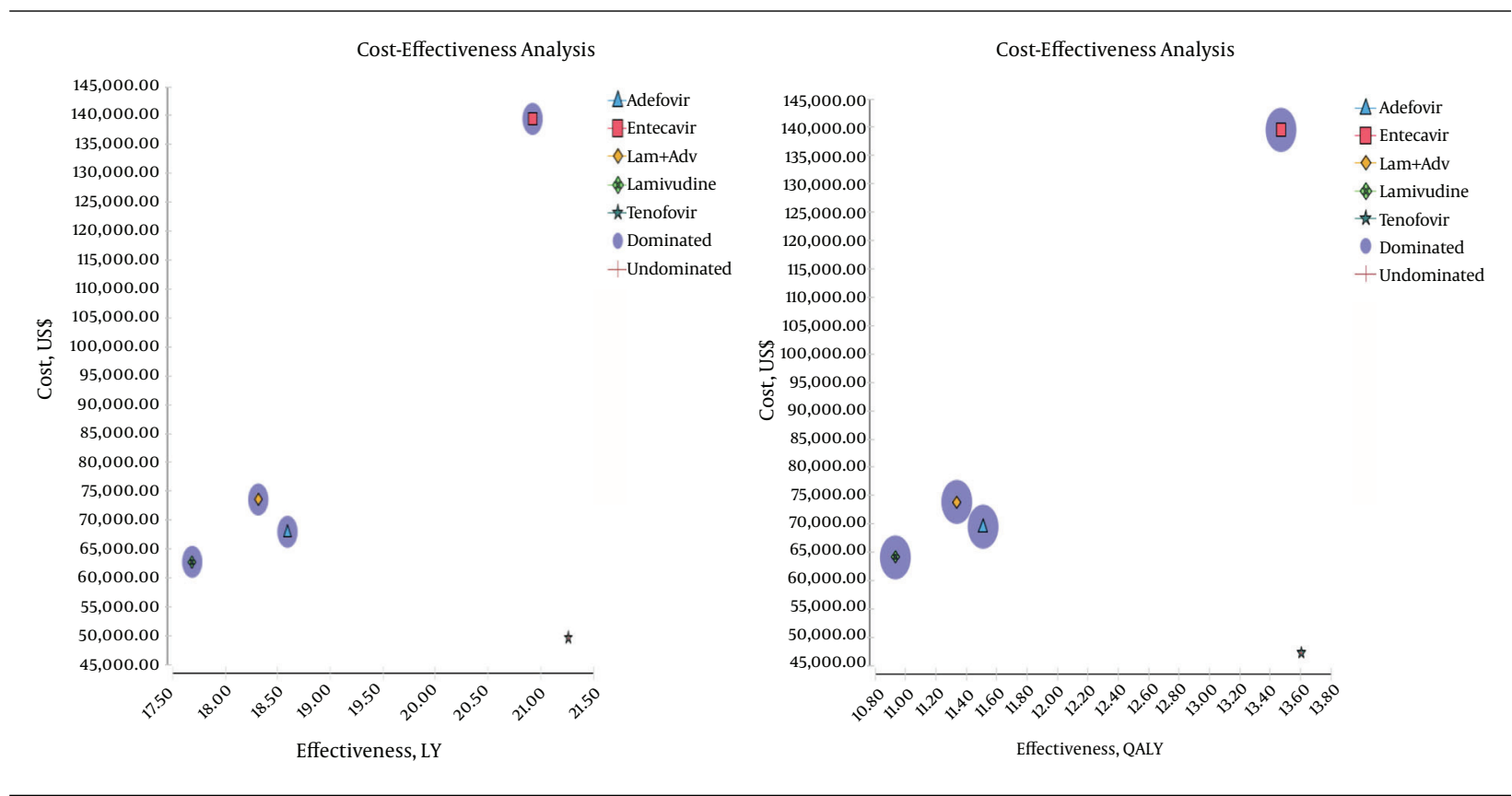

Figure 2. Comparison of the Cost-Effectiveness of the Five Drug Strategies in Patients With HBeAg-Negative CHB Based on LYG and QALY

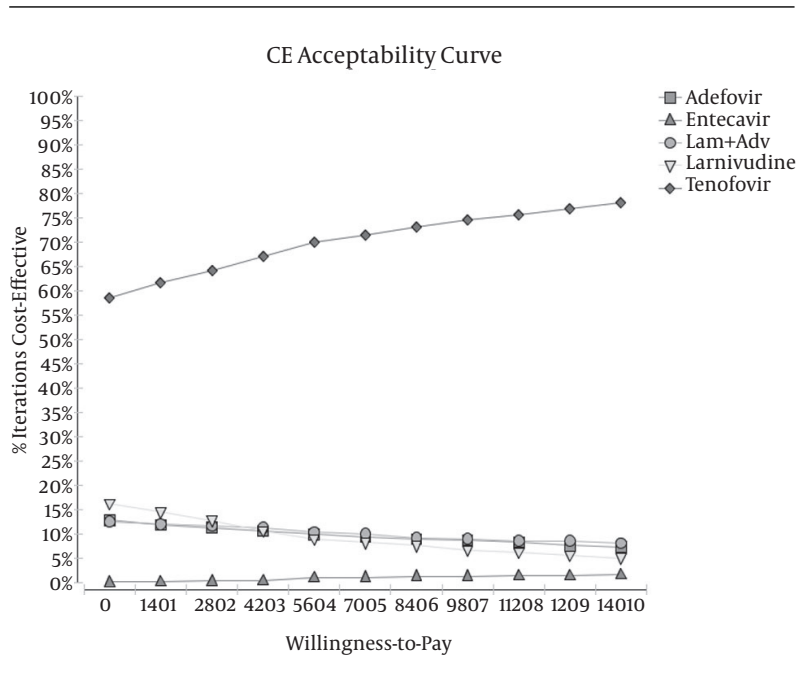

Figure 3. Cost-Effectiveness Acceptability Curve Obtained Through a Monte Carlo Simulation

strategies and that it achieved the best results. It also confirmed that TDF was superior to all the other drug treatment strategies. As the results of the PSA did not alter the status of TDF as the most effective treatment, the results of the present study seem to be robust. The findings of the current study are in line with those of studies by He et al. in Canada, Wiens in Brazil, and Tantai et al. in Thailand (12, $16,58)$.
The cost-effectiveness acceptability curve, which was the output curve of PSA, also showed that TDF was the most cost-effective therapy in 59\% - 78\% of simulations in HBeAgnegative cases for thresholds below $\$ 14010$. The results of this study are consistent with those an earlier study by $\mathrm{He}$ al. (12), who reported that TDF was the most effective strategy in $65 \%-78 \%$ of cases.

Furthermore, as shown by the results of the incremental cost-effectiveness scatter plot, which was another output of the PSA, in most cases, compared with the other alternatives, TDF was in an acceptable area below the threshold. In patients with HBeAg-negative $\mathrm{CHB}$, compared with the alternatives (LAM, ADV, ETV, and ADV + LAM), TDF was in acceptable area below the threshold in $85 \%, 84 \%, 62 \%$, and $83 \%$ of cases and was superior to the other strategies. The results of the present study are in line with those of He et al. (12).

In addition, the results obtained from the estimation of QALY and cost savings associated with the use of TDF in HBeAg-negative patients compared with the alternatives showed that it reduced the progress of the disease and serious complications in the future. In addition, it led to cost savings and a very significant increase in the number of QALYs. The results of this study are consistent with those of studies conducted by Jing et al. and Tantai et al. $(12,58)$. TDF seems to be the optimal drug regimen to stop or slow the progression of liver disease. Therefore, TDF, which was identified as the most cost-saving strategy in this study and 
Figure 4. Incremental Cost-Effectiveness of TDF Compared With the Other Antivirals (ADV, LAM, LAM + ADV, and ETV)

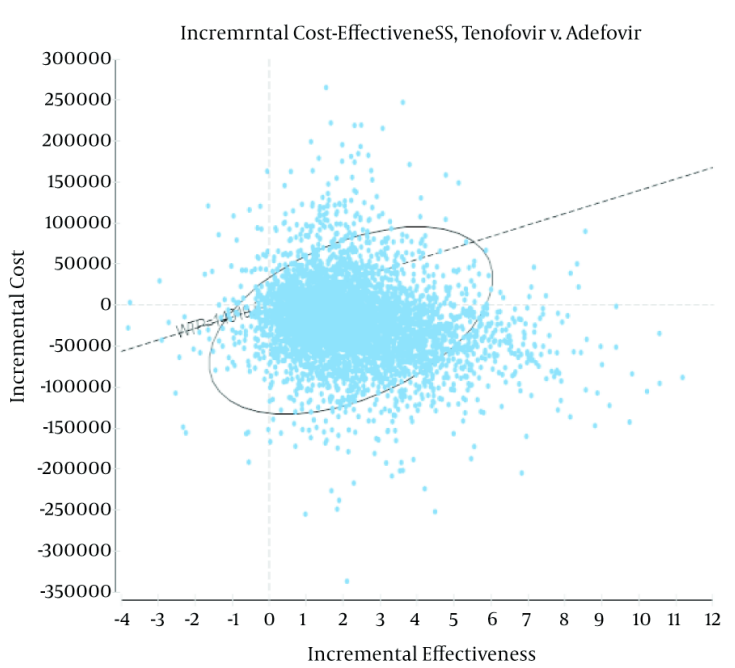

Incremrntal Cost-EffectiveneSS, Tenofovir v. Lam+Adv

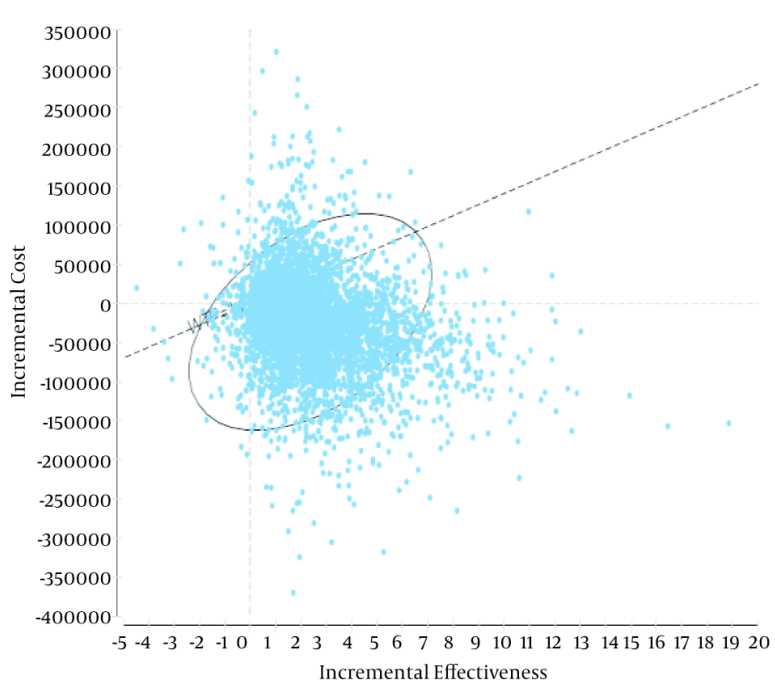

Incremrntal Cost-EffectiveneSS, Tenofovir v. Lamivudine

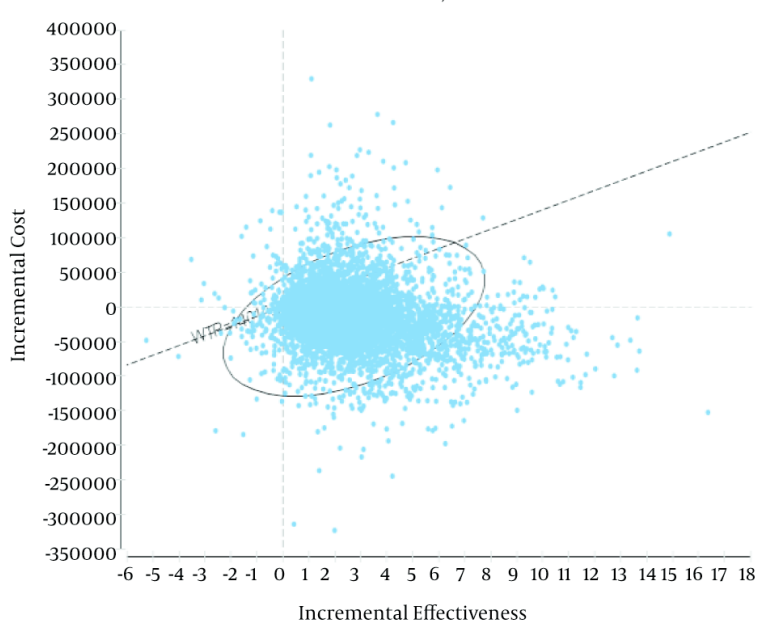

Incremrntal Cost-EffectiveneSS, Tenofovir v. Entecavir

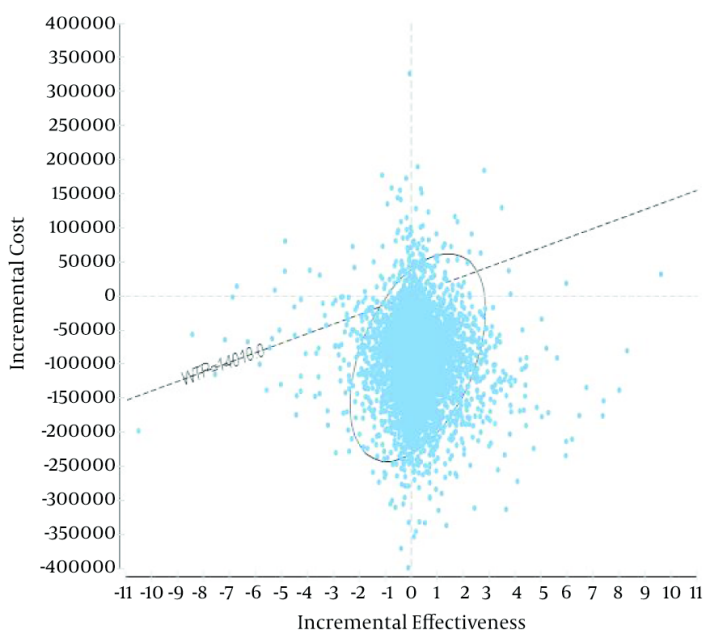

The scatter plots show the difference in cost and QALY results of 5000 simulations.

some previously published studies $(12,16,58)$, should be the first choice in cases of LAM or ADV drug resistance.

Most of the drugs in the present study are manufactured in Iran and therefore do not require high expenditures by either patients or the health system. However, given the better response of TDF compared with the other drugs, it can prevent and reduce high costs associated with complications (i.e., cirrhosis and cancer) that emerge in severe stages of the disease. A regular review of the literature after the introduction of new drugs can ensure that the dominant drugs are excluded and replaced by the domi- nant drugs. This will benefit all those involved in the health system (i.e., the government, insurance industry, and patients).

The strengths of this study are as follows: The model included both direct medical and various indirect nonmedical costs, and it employed the Monte Carlo Markov method for microsimulation modeling. In addition, the data on the costs and effectiveness were collected locally and based on observations, patients' self-reports, and experts' opinions. Furthermore, the values of the country were used for the first time to convert the 5-digit code numbers of the 
questionnaire to numerical utility. An additional strength of the model used in the study was the ability to switch to another drug therapy for those patients who gave up drug therapy. This ability distinguishes the present model from models used in previous studies. Finally, for the first time, a microsimulation approach was used to assess the costeffectiveness of antiviral drugs in patients with chronic HBV infection.

One of the limitations of this study was the absence of data in some cases, especially data on the transitional probabilities of the disease. Hence, a constant rate was used in this study. Although the results showed that TDF was the superior and most cost-effective option for HBeAg-negative patients, to generalize the results to other settings, various factors could influence the findings. These include the epidemiology of the disease, demographics of the study population, resource availability, costs, valuing outcomes by individuals, thresholds, and the use of indicators of effectiveness. However, as this study included a range of important features of the patients, including age, sex, and duration of the disease, and the data were extracted from several main referral centers in the country, many of the results can likely be generalized.

In conclusion, according to the results of this study, among nucleos $(t)$ ide analogues evaluated, TDF was the optimal and most cost-effective strategy for patients with CHB. Hence, it can be used as first-line therapy and for patients switching from other drugs. Furthermore, as TDF can reduce treatment times and manage drug resistance, TDF can be recommended as first-line therapy for patients with CHB.

\section{Acknowledgments}

This study was part of a PhD thesis in the field of pharmacoeconomics and pharmaceutical administration in the faculty of pharmacy, Tehran University of Medical Sciences. The authors would like to thank the Baqiyatallah research center for gastroenterology and liver diseases, Shiraz University of Medical Sciences, and the center for development of clinical research of Nemazee hospital. We would also like to thank Dr. Nasrin Shokrpour for editorial assistance and Sheida Ebrahimi and Maryam Gholami for their cooperation in the research.

\section{Footnote}

Authors' Contribution: Khosro Keshavarz: gathering the data, analyzing the collected data, and contributing to the design of the research method; Abbas Kebriaeezadeh, Seyed Moayed Alavian, and Shekoufeh Nikfar: designing the research method, revising the manuscript, and final approval of the data; Ali Akbari Sari and Farhad Lotfi: designing the research method and revising the manuscript; Maryam Keshvari, Mohsen Rezaei Hemami, Amir Hashemi Meshkini, and Mehdi Javanbakht: data gathering and drafting the manuscript; All the authors read and approved the final manuscript.

\section{References}

1. Lok AS. Chronic hepatitis B. N Engl J Med. 2002;346(22):1682-3. doi: 10.1056/NEJM200205303462202. [PubMed:12037146].

2. Alavian SM, Fallahian F, Lankarani KB. The changing epidemiology of viral hepatitis B in Iran. J Gastrointestin Liver Dis. 2007;16(4):403-6. [PubMed: 18193122]

3. Lee WM. Hepatitis B virus infection. NEngl J Med. 1997;337(24):1733-45 doi: 10.1056/NEJM199712113372406. [PubMed: 9392700].

4. Lai CL. Chronic hepatitis B in Hong Kong: immunization strategies for the control of hepatitis B virus infection. Hepatitis B Asian-Pacific region. 1997;1:79-87.

5. Keshavarz K, Kebriaeezadeh A, Alavian SM, Akbari Sari A, Abedin Dorkoosh F, Keshvari M, et al. Economic burden of hepatitis B virusrelated diseases: evidence from iran. Hepat Mon. 2015;15(4):25854. doi: 10.5812/hepatmon.15(4)2015.25854. [PubMed: 25977694].

6. Alavian SM, Hajarizadeh B, Ahmadzad-Asl M, Kabir A, BagheriLankarani K. Hepatitis B Virus infection in Iran: A systematic review. Hepat Mon. 2008;8(4):281-94.

7. Iran Statistic Centre . Iran: Iran Statistic Centre; 2015. [updated 2015] Available from: http://www.amar.org.ir.

8. Alavian SM, Keyvani H, Rezai M, Ashayeri N, Sadeghi HM. Preliminary report of hepatitis B virus genotype prevalence in Iran. World J Gastroenterol. 2006;12(32):5211-3. [PubMed: 16937535].

9. Haghshenas MR, Arabi M, Mousavi T. Hepatitis B genotypes in iran. Mater Sociomed. 2014;26(2):129-33. doi: 10.5455/msm.2014.26.129-133. [PubMed: 24944540].

10. Mohamadnejad M, Montazeri G, Fazlollahi A, Zamani F, Nasiri J, Nobakht $\mathrm{H}$, et al. Noninvasive markers of liver fibrosis and inflammation in chronic hepatitis B-virus related liver disease. Am J Gastroenterol. 2006;101(11):2537-45. doi: 10.1111/j.1572-0241.2006.00788.x. [PubMed: 17029616].

11. Sherman M, Shafran S, Burak K, Doucette K, Wong W, Girgrah N, et al. Management of chronic hepatitis B: consensus guidelines. Can J Gastroenterol. 2007;21 Suppl C:5-24. [PubMed: 17568823].

12. He J, Bowen JM, Xie F, Goeree R. Cost-effectiveness analysis of antiviral treatments for HBeAg-positive chronic hepatitis B in Canada. Value Health. 2012;15(6):894-906. doi: 10.1016/j.jval.2012.06.005. [PubMed: 22999140].

13. Dakin H, Bentley A, Dusheiko G. Cost-utility analysis of tenofovir disoproxil fumarate in the treatment of chronic hepatitis B. Value Health 2010;13(8):922-33. doi: 10.1111/j.1524-4733.2010.00782.x. [PubMed: 20825619].

14. Yuan Y, Iloeje UH, Hay J, Saab S. Evaluation of the cost-effectiveness of entecavir versus lamivudine in hepatitis BeAg-positive chronic hepatitis B patients. J Manag Care Pharm. 2008;14(1):21-33. doi: 10.18553/jmcp.2008.14.1.21. [PubMed: 18240879].

15. Earnshaw J, Lewis G. NICE Guide to the Methods of Technology Appraisal: pharmaceutical industry perspective. Pharmacoeconomics. 2008;26(9):725-7. [PubMed:18767892].

16. Wiens A, Venson R, Correr CJ, Pontarolo R. Cost-effectiveness of telbivudine versus lamivudine for chronic hepatitis B. Braz J Infect Dis. 2011:15(3):225-30. [PubMed: 21670922].

17. Poorolajal J, Majdzadeh R. Prevalence of chronic hepatitis B infection in Iran: a review article. J Res Med Sci. 2009;14(4):249-58. [PubMed: 21772891]. 
18. Salem F, Hekmat S, Aghasadeghi MR, Javadi F, Gholami H, Mostafavi E. Prevalence and Risk Factors of Hepatitis B Virus Genotype D Amongst Inmates in Alborz Province, Iran: A Cross-Sectional Survey. JundishapurJMicrobiol. 2013;6(6) doi: 10.5812/jjm.10221.

19. Abdoli G. Estimating the social discount rate for Iran [in Persian]. J Econ Res. 2009;3:135-56.

20. Robberstad B. Estimation of private and social time preferences for health in northern Tanzania. Soc Sci Med. 2005;61(7):1597-607. doi: 10.1016/j.socscimed.2005.03.013. [PubMed: 15885866].

21. Nikfar S, Kebriaeezadeh A, Dinarvand R, Abdollahi M, Sahraian MA Henry D, et al. Cost-effectiveness of different interferon beta products for relapsing-remitting and secondary progressive multiple sclerosis: Decision analysis based on long-term clinical data and switchable treatments. Daru. 2013;21(1):50. doi:10.1186/2008-2231-21-50. [PubMed: 23800250].

22. Mohammadnezhad M, Malekzadeh Z. Guidelines On The Management Of Chronic Hepatitis B [in Persian]. Govaresh J. ;19(2):128-40.

23. Bobes J, Canas F, Rejas J, Mackell J. Economic consequences of the adverse reactions related with antipsychotics: an economic model comparing tolerability of ziprasidone, olanzapine, risperidone, and haloperidol in Spain. Prog Neuropsychopharmacol Biol Psychiatry. 2004;28(8):1287-97. doi: 10.1016/j.pnpbp.2004.06.017. [PubMed: 15588755].

24. Bell C, Graham J, Earnshaw S, Oleen-Burkey M, Castelli-Haley J, Johnson K. Cost-effectiveness of four immunomodulatory therapies for relapsing-remitting multiple sclerosis: a Markov model based on long-term clinical data. J Manag Care Pharm. 2007;13(3):245-61. doi: 10.18553/jmcp.2007.13.3.245. [PubMed: 17407391].

25. Nuijten MJ, Hutton J. Cost-effectiveness analysis of interferon beta in multiple sclerosis: a Markov process analysis. Value Health. 2002;5(1):44-54. doi: 10.1046/j.1524-4733.2002.51052.x. [PubMed: 11873383].

26. Nikfar S. A new model for decision analysis in economic evaluations of switchable health interventions. J Med Hypotheses Ideas. 2012;6(1):12-5. doi: 10.1016/j.jmhi.2012.03.008

27. Lok AS, Zoulim F, Locarnini S, Bartholomeusz A, Ghany MG, Pawlotsky JM, et al. Antiviral drug-resistant HBV: standardization of nomenclature and assays and recommendations for management. Hepatology. 2007;46(1):254-65. doi:10.1002/hep.21698. [PubMed: 17596850].

28. Zoulim F, Locarnini S. Hepatitis $B$ virus resistance to nucleos(t)ide analogues. Gastroenterology. 2009;137(5):1593-608. doi: 10.1053/j.gastro.2009.08.063. [PubMed:19737565]

29. Pawlotsky JM, Dusheiko G, Hatzakis A, Lau D, Lau G, Liang T], et al. Virologic monitoring of hepatitis B virus therapy in clinical trials and practice: recommendations for a standardized approach Gastroenterology. 2008;134(2):405-15. doi:10.1053/j.gastro.2007.11.036 [PubMed: 18242209].

30. European Association For The Study Of The Liver . EASL clinical practice guidelines: Management of chronic hepatitis B virus infection. J Hepatol. 2012;57(1):167-85. doi: 10.1016/j.jhep.2012.02.010. [PubMed: 22436845].

31. Gish R, Jia JD, Locarnini S, Zoulim F. Selection of chronic hepatitis B therapy with high barrier to resistance. Lancet Infect Dis. 2012;12(4):341-53. doi: 10.1016/S1473-3099(11)70314-0. [PubMed 22326017].

32. Berg T, Möller B, Gerken G, Jung MC, Spengler U, Hartmann H, et al. Tenofovir disoproxil fumarate(TDF) versus Emtricitabine plus TDF for treatment of chronic Hepatitis B (CHB) in subjects with persistent viral replication receiving Adefovir dipivoxil (ADV). Zeitschrift Gastroenterol. 2008;46(9) doi:10.1055/s-0028-1089367.

33. Sung JJY, Lai JY, Zeuzem S, Chow WC, Heathcote E, Perrillo R, et al. A randomised double-blind phase II study of lamivudine (LAM) compared to lamivudine plus adefovir dipovoxil (ADV) for treatment naïve patients with chronic hepatitis B (CHB): Week 52 analysis. J Hepatol. 2003;38:25-6. doi: 10.1016/s0168-8278(03)80485-3.

34. Lai CL, Leung N, Teo EK, Tong M, Wong F, Hann HW, et al. A 1-year trial of telbivudine, lamivudine, and the combination in patients with hepatitis B e antigen-positive chronic hepatitis B. Gastroenterology. 2005;129(2):528-36. doi: 10.1016/j.gastro.2005.05.053. [PubMed: 16083710].

35. Woo G, Tomlinson G, Nishikawa Y, Kowgier M, Sherman M, Wong DK, et al. Tenofovir and entecavir are the most effective antiviral agents for chronic hepatitis B: a systematic review and Bayesian meta-analyses. Gastroenterology. 2010;139(4):1218-29. doi: 10.1053/j.gastro.2010.06.042. [PubMed: 20600036].

36. Dakin H, Fidler C, Harper C. Mixed treatment comparison metaanalysis evaluating the relative efficacy of nucleos(t)ides for treatment of nucleos(t)ide-naive patients with chronic hepatitis B. Value Health. 2010;13(8):934-45. doi:10.1111/j.1524-4733.2010.00777.x. [PubMed: 20825624].

37. Marcellin P, Heathcote EJ, Buti M, Gane E, de Man RA, Krastev Z, et al. Tenofovir disoproxil fumarate versus adefovir dipivoxil for chronic hepatitis B. N Engl J Med. 2008;359(23):2442-55. doi: 10.1056/NEJMoa0802878. [PubMed: 19052126].

38. Iloeje UH, Yang HI, Su J, Jen CL, You SL, Chen CJ, et al. Predicting cirrhosis risk based on the level of circulating hepatitis B viral load. Gastroenterology. 2006;130(3):678-86. doi: 10.1053/j.gastro.2005.11.016. [PubMed: 16530509].

39. Chang TT, Gish RG, de Man R, Gadano A, Sollano J, Chao YC, et al. A comparison of entecavir and lamivudine for HBeAg-positive chronic hepatitis B. N Engl J Med. 2006;354(10):1001-10. doi: 10.1056/NEJMoa051285. [PubMed: 16525137].

40. Eun JR, Lee HJ, Kim TN, Lee KS. Risk assessment for the development of hepatocellular carcinoma: according to on-treatment viral response during long-term lamivudine therapy in hepatitis B virus-related liver disease. J Hepatol. 2010;53(1):118-25. doi: 10.1016/j.jhep.2010.02.026. [PubMed: 20471129].

41. Yang HI, Yuen MF, Chan HL, Han KH, Chen PJ, Kim DY, et al. Risk estimation for hepatocellular carcinoma in chronic hepatitis B (REACHB): development and validation of a predictive score. Lancet Oncol. 2011;12(6):568-74. doi: 10.1016/S1470-2045(11)70077-8. [PubMed: 21497551].

42. Toy M, Onder FO, Idilman R, Kabacam G, Richardus JH, Bozdayi M, et al. The cost-effectiveness of treating chronic hepatitis B patients in a median endemic and middle income country. Eur J Health Econ. 2012;13(5):663-76. doi: 10.1007/s10198-012-0413-8. [PubMed: 22815098].

43. Yurdaydin C, Bozkaya H, Cetinkaya H, Sahin T, Karaoguz D, Toruner $\mathrm{M}$, et al. Lamivudine vs lamivudine and interferon combination treatment of HBeAg(-) chronic hepatitis B. J Viral Hepat. 2005;12(3):262-8. doi: 10.1111/j.1365-2893.2005.00566.x. [PubMed: 15850466].

44. Chan HL, Heathcote EJ, Marcellin P, Lai CL, Cho M, Moon YM, et al. Treatment of hepatitis $\mathrm{B}$ e antigen positive chronic hepatitis with telbivudine or adefovir: a randomized trial. Ann Intern Med. 2007;147(11):745-54. [PubMed: 17909201].

45. Levy AR, Kowdley KV, Iloeje U, Tafesse E, Mukherjee J, Gish R, et al. The impact of chronic hepatitis B on quality of life: a multinational study of utilities from infected and uninfected persons. Value Health. 2008;11(3):527-38. doi: 10.1111/j.1524-4733.2007.00297.x. [PubMed: 18179664].

46. Chen CJ, Yang HI, Iloeje UH, Reveal-Hbv Study Group . Hepatitis B virus DNA levels and outcomes in chronic hepatitis B. Hepatology. 2009;49(5 Suppl):72-84. doi: 10.1002/hep.22884. [PubMed: 19399801].

47. Kanwal F, Gralnek IM, Martin P, Dulai GS, Farid M, Spiegel BM. Treatment alternatives for chronic hepatitis B virus infection: a costeffectiveness analysis. Ann Intern Med. 2005;142(10):821-31. [PubMed: 15897532].

48. Lynch SV, Balderson GA. 2007.

49. Sali S, Alavian SM, Foster GR, Keyvani H, Mehrnoosh L, Mohammadi N. Influencing Factors on the Outcome and Prognosis of Patients With HBV Infction: Seven Years Follow-up. Hepat Mon. 2013;13(7):8743. doi: 10.5812/hepatmon.8743. [PubMed: 24066002]. 
50. WHO . Life tables by country Iran (Islamic Republic of) Geneva: World Health Organization; Available from: http://apps.who.int/gho/data/ view.main.60760.

51. Duberg AS, Torner A, Davidsdottir L, Aleman S, Blaxhult A, Svensson A, et al. Cause of death in individuals with chronic HBV and/or $\mathrm{HCV}$ infection, a nationwide community-based register study. $\mathrm{J}$ Viral Hepat. 2008;15(7):538-50. doi: 10.1111/j.1365-2893.2008.00982.x. [PubMed: 18397223].

52. Sun X, Faunce T. Decision-analytical modelling in health-care economic evaluations. Eur J Health Econ. 2008;9(4):313-23. doi: 10.1007/s10198-007-0078-x. [PubMed:17943332].

53. Goudarzi R. Estimating quality weights for EQ-5D health states with the time trade-off (TTO) method in Iran [Dissertation]. Iran: Tehran University of Medical Sciences; 2014.

54. Central Bank of the Islamic Republic of Iran . The Rate Currency Iran: Central Bank of the Islamic Republic of Iran; Available from: http://
www.cbi.ir/ExRates/rates_fa.aspx.

55. World Bank. PPP conversion factor, private consumption (LCU per international \$) US: The World Bank; Available from: http://www.cbi.ir/ ExRates/rates_fa.aspx.

56. Buti M, Brosa M, Casado MA, Rueda M, Esteban R. Modeling the cost-effectiveness of different oral antiviral therapies in patients with chronic hepatitis B. J Hepatol. 2009;51(4):640-6. doi: 10.1016/j.jhep.2009.04.013. [PubMed: 19576651].

57. Shillcutt SD, Walker DG, Goodman CA, Mills AJ. Cost effectiveness in low- and middle-income countries: a review of the debates surrounding decision rules. Pharmacoeconomics. 2009;27(11):903-17. doi: 10.2165/10899580-000000000-00000. [PubMed: 19888791].

58. Tantai N, Chaikledkaew U, Tanwandee T, Werayingyong P, Teerawattananon Y. A cost-utility analysis of drug treatments in patients with HBeAg-positive chronic hepatitis B in Thailand. BMC Health Serv Res. 2014;14:170. doi: 10.1186/1472-6963-14-170. [PubMed: 24731689]. 